\title{
EXPERIMENTAL STUDY ON TILTING STIFFNESS OF OIL HYDROSTATIC SHALLOW RECESS THRUST BEARINGS
}

\author{
F. A. Tripkewitz ${ }^{1,2 *}$, M. Fritz ${ }^{1}$, M. Weigold ${ }^{2}$ \\ ${ }^{1} \mathrm{KERN}$ Microtechnik GmbH, Eschenlohe, Germany \\ ${ }^{2}$ Technische Universität Darmstadt, Institute of Production Management, Technology and Machine Tools (PTW), \\ Darmstadt, Germany \\ ${ }^{*}$ Corresponding author; e-mail: fabian.tripkewitz@kern-microtechnik.com
}

\begin{abstract}
Circular shallow recess bearings generate a not rotationally symmetrical pressure profile if they are not aligned parallel. The throttling effect of the shallow recess makes this response possible. Furthermore, the generated pressure profile acts against the tilting. As a result, a shallow recess bearing generates a tilting stiffness. This article outlines an experimental approach to characterize the tilting stiffness of an oil hydrostatic shallow recess thrust bearing with micro step and gap height. For this purpose, we developed a small test bench. The measured tilting stiffnesses are compared with a numerical solution based on the 2D-Reynolds equation.
\end{abstract}

\section{Keywords:}

shallow step; inherently compensated; hydrostatic bearing; angular stiffness; 2D-Reynolds

\section{INTRODUCTION}

Machine tools have been using oil hydrostatics for decades. Hydrostatic bearings have almost no wear and therefore constant properties. Additionally, high stiffness, viscous damping and high error averaging predestinate them for high precision machines. [Liu 2017]

A single deep recess hydrostatic pocket can only support coaxial compressive forces due to negligible tilting stiffness [Rowe 2012]. Therefore, bearing units consist of several pockets to carry eccentric loads. The use of multiple pockets requires a hydraulic decoupling. External devices, which can either have a constant or variable resistance, usually accomplish this task. In addition, for high natural frequencies, the compressible volume of the fluid between the device and the pocket must be reduced [Pollmann 1989]. This requirement restricts the placement of the external devices and thus the available space. In 1958, C. R. Adams described a different concept for air bearings, in which the bearing geometry includes the hydraulic decoupling [Adams 1958]. This characteristic eliminates the need for external controllers. Furthermore, the resulting pressure profile is dependent on tilt, as shown in Fig. 1. The corresponding torque counteracts the tilt, which results in a tilting stiffness for a single bearing. Donaldson investigated the behavior with incompressible fluid for shallow recess journal bearings [Donaldson 1965]. Further experimental studies validate the 2D Reynolds equation as an approximation for shallow recess journal bearings [Carpenter 1969].
Knapp measured the tilting stiffness of a bearing unit based on step compensation with small gap height, fed with compressed air [Knapp 2019]. Furthermore, Van Beek presented dimensionless design parameters for aerostatic shallow recess thrust bearings [Beek 2019]. These describe the theoretical self-aligning capability of a thrust bearing.

This paper investigates the tilting stiffness of shallow recess thrust bearings at small gap heights $(10 \mu \mathrm{m})$ and high supply pressure $(10 \mathrm{MPa})$ theoretically and experimentally. We designed a test bench to measure the tilting stiffness of different bearing geometries with low measurement uncertainty. The included graphs show the measured tilting stiffness up to large angular changes. In addition, an approximation of constant tilting stiffness for small tilting angles is proposed.
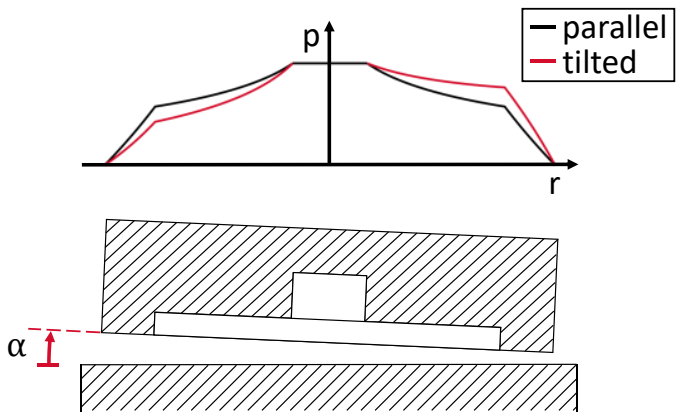

Fig. 1: Influence of tilt on pressure distribution. 


\section{THEORY}

For approximating the resulting pressure distribution of a thin lubricating oil film, the 2D-Reynolds equation is often used [Bassani 1992]. For a stationary, rotationally symmetrical bearing with no relative velocity of the bearing components, the formula is as follows:

$\frac{\partial}{\partial r}\left(r h^{3} \frac{\partial p}{\partial r}\right)+\frac{1}{r} \frac{\partial}{\partial \theta}\left(h^{3} \frac{\partial p}{\partial \theta}\right)=0$

The discontinuity of the film height at the ideal step contradicts the assumption of laminar flow. In reality, however, the transition is continuous due to the corner radius of the tool. Therefore, the assumption of laminar flow is acceptable.

Fig. 2 shows a schematic bearing geometry and its nomenclature.

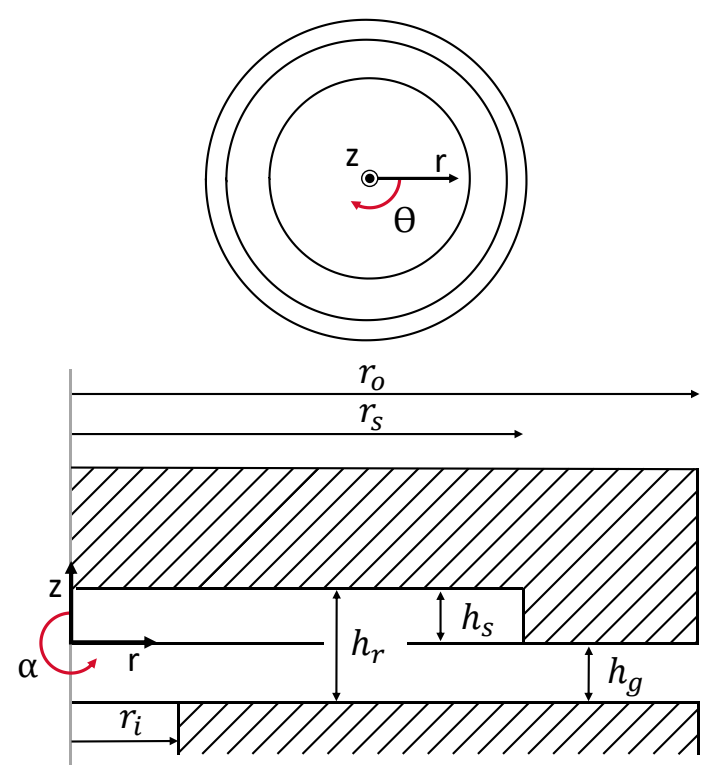

Fig. 2: Nomenclature of the shallow recess bearing geometry.

Three areas which form passages with different heights, define the working principle. Pressurized oil flows through the inner bearing radius $r_{i}$ into the recess with height $h_{r}$. At the step radius $r_{s}$ the channel height reduces by step height $h_{s}$ to gap height $h_{g}$. Ambient pressure is present at the outer bearing radius $r_{o}$.

The calculation of the resulting gap height $h$ at an angular change requires a center of rotation. In the following, the center of rotation corresponds to the origin in Fig. 2.

$h= \begin{cases}h_{r}+r \cos (\theta) \tan (\alpha) & \text { for } r_{i} \leq r \leq r_{s} \\ h_{g}+r \cos (\theta) \tan (\alpha) & \text { for } r_{s}<r \leq r_{o}\end{cases}$

Since there is no analytical solution for Eq. (1), it is solved numerically by the finite difference method (FDM). The result represents the pressure profile $p(r, \theta)$. Eq. (3) provides the corresponding reaction torque $M_{\alpha}$ for an angular error of $\alpha$.

$$
M_{\alpha}=\int_{0}^{2 \pi} \int_{r_{i}}^{r_{o}} r^{2} p(r, \theta) \cos (\theta) d r d \theta
$$

The simplified description of the bearing geometry depends on three dimensionless parameters. These consist of the dimensionless land to recess ratio $L$, the height ratio $H$ and the radius ratio $R$. These three parameters fully define the bearing geometry if no angular error exists.

$L=\frac{r_{s}-r_{i}}{r_{o}-r_{i}}$

$H=\frac{h_{g}}{h_{s}}$

$R=\frac{r_{i}}{r_{o}}$

For parallel aligned surfaces, a combination of $L, H$ and $R$ defines the relative pressure distribution $p(r, \theta)$.

The conversion of the dimensionless parameters to the tilting stiffness $k_{M}$ of an explicit geometry requires a scaling factor: there is a direct proportionality to the supply pressure $p_{s}$ and the third power of bearing diameter $D$. Further, the tilting stiffness is inversely proportional to the initial gap height $h_{g}$

$k_{M} \sim \frac{p_{s} D^{3}}{h_{g}}$

Based on Eq. (4) - (7), the gap height $h_{g}$ influences the pressure profile as well as the scaling factor. The same applies to the bearing diameter $D$. A variation of the inner radius $r_{i}$, the step radius $r_{s}$ and the step height $h_{s}$ can eliminate the scaling factor in measurements.

\section{EXPERIMENTAL SETUP}

For the experimental investigation of the tilting stiffness of bearing geometries with different parameters $L, H$ and $R$, we developed a test bench. Fig. 3 shows an overview.

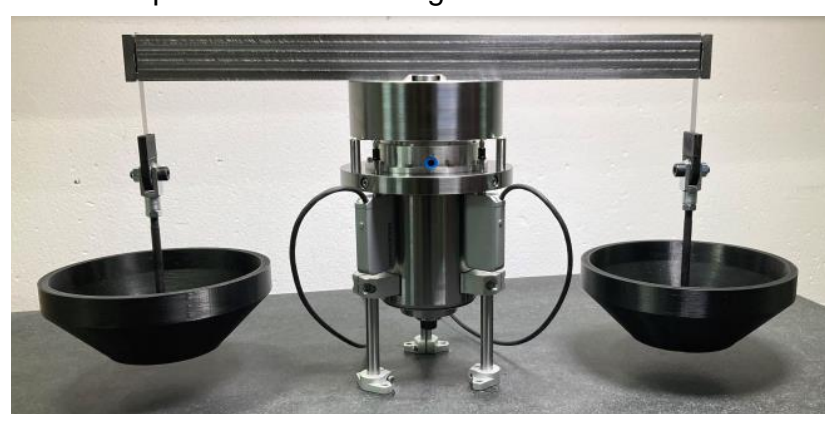

Fig. 3: Overview of the experimental setup.

\subsection{Bearing unit}

The central element of the test bench is the bearing unit. Fig. 4 displays its sectional view. The main design criterion was the reduction of random errors in the measurements.

The bearing module (1) represents the bearing geometry, including the shallow recess. Beneath that, the main body (2) represents the flat mating surface. A flexure (3) secures the bearing module relative to the main body without restricting the movement in both tilting and the z-direction. By mounting the flexure in the plane of the bearing, locating forces do not cause tilting moments approximately. 


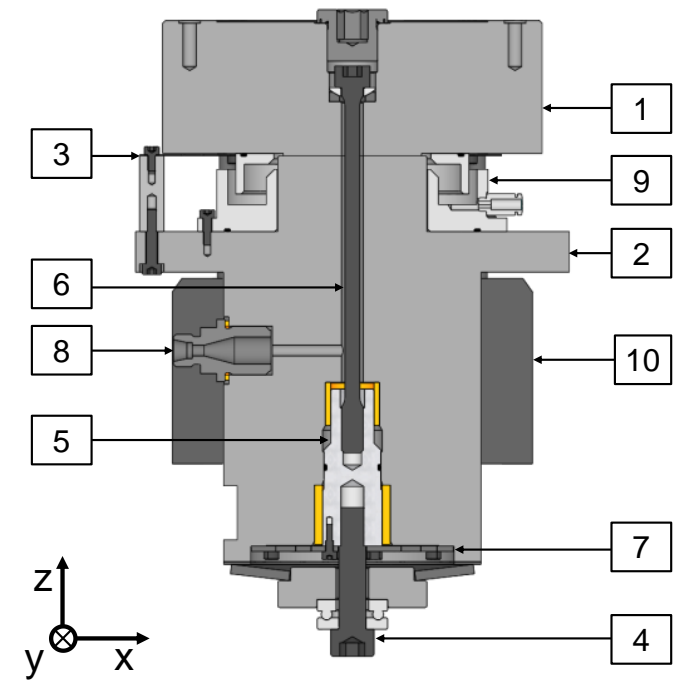

Fig. 4: Bearing unit cross-section.

A screw (4) rests on a thrust ball bearing and applies the preload of the pocket module. The installed disc spring increases the compliance and allows in combination with the ball bearing finer adjustments. A guided coupling (5) and an expansion screw (6) transmit the force to the bearing module. The double logarithmic spiral slitted flexure (7) restricts the coupling's rotational degree of freedom and therefore the transmission of rotation of the preload screw to the bearing module. To compensate for manufacturing tolerances, the head of the expansion screw rests on a spherical washer in a conical seat. Via a hydraulic connector (8), the pressurized oil flows radially into the main body, around the expansion screw and enters the bearing. A gutter (9) collects the leaving oil and returns it to the oil tank. Bushings in the main body clamp the shafts of the four displacement transducers (10). Their tips rest on the target surfaces of the bearing module. Machining the target surfaces and the land of the bearing in one plane avoids second-order Abbe errors.

A finite element analysis was carried out to estimate the tilting stiffness of the test bench. For this, the simulation model only considers components that connect the bearing module with the main body. These include the expansion screw and the flexure. Fig. 5 summarizes the corresponding simulation model and the simulation result. Since the friction conditions of the coupling are unknown, the simulation assumes a fixed support of the thread (B). For the three holes of the flexure (C), the same boundary condition applies. The simulation further assumes a bonded contact for all present interfaces. The first contact is between the bolt head and the bearing module. Here, the model neglects the spherical washer and the conical seat. The second contact is between the bearing module and the flexure. Here, the contact area is limited to the diameter of the bolt heads. A moment of $0.1 \mathrm{Nm}$ acts to the bearing surface $(A)$.

The simulation shows a relative displacement of the target surfaces of $55.8 \mu \mathrm{m}$. Over a distance of $130 \mathrm{~mm}$, this is equivalent to $429.2 \mu \mathrm{rad}$, which corresponds to a tilting stiffness of $0.23 \mathrm{Nm} / \mathrm{mrad}$.

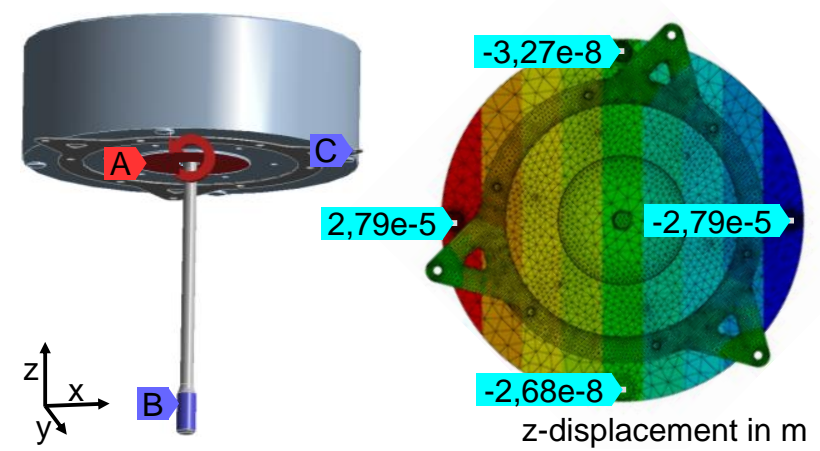

Fig. 5: Model to simulate the tilting stiffness of the test bench (left) and the corresponding displacement (right).

\subsection{Application of torque}

The mechanical contraption for precise torque application follows the calibration bench of the PTB [Schlegel 2018]. Its main component is a lever of known length $l_{\text {lever }}$. At each end, brackets clamp a thin feeler gauge strip of thickness $h_{f}$ against the faces of the lever. Thereby, forces introduced have a defined distance. Fig. 6 provides a schematic overview of the contraption.

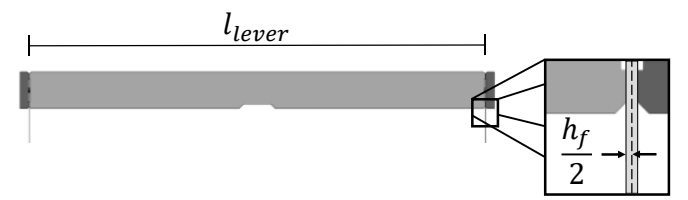

Fig. 6: Nomenclature torque contraption.

Assuming that the force acts at half the feeler gauge thickness $h_{f}$, the resulting lever $l_{\text {res }}$ is calculated by:

$l_{\text {res }}=l_{\text {lever }}+h_{f}$

Masses with defined weights represent the incremental loads. By multiplying the mass with the gravitational acceleration $g$, the corresponding gravitational force results. The weights are subtracted from one side and added to the other side. This procedure ensures that the axial load remains constant. The equivalent torque $M$ by moving a mass $m$ is calculated by:

$M=m g l_{\text {res }}$

\subsection{Measurement of displacement and tilting}

The test bench contains four displacement transducers positioned every $90^{\circ}$ with a radial distance of $65 \mathrm{~mm}$ from the bearing center. The greater distance compared to the bearing diameter allows a mechanical increase of sensitivity. However, elastic and thermoelastic effects increase with greater distance. The measured data of the displacement transducers contains information on the axial displacement and the tilting. Section 5.1 describes the exact procedure of calculation.

Resolving $0.1 \mu \mathrm{rad}$ at a distance of $65 \mathrm{~mm}$ requires a resolution of $6.5 \mathrm{~nm}$. For this reason, the test bench utilizes four Heidenhain MT 1281 displacement transducers in combination with a Heidenhain EIB 741. 


\subsection{Measurement uncertainty}

Due to the small displacements present, the displacement transducers primarily influence the measurement uncertainty.

The following estimation of the measurement uncertainty refers to a $10 \mu \mathrm{rad}$ angular change and a coverage factor $k=2$. With this, the uncertainty of the angle is $6.15 \%$ and the calculated tilting stiffness has an uncertainty of $12.3 \%$.

\subsection{Bearing dimensions}

The study includes nine different bearing modules, M1 - M9, with varying bearing dimensions. Tab. 1 lists the parameters $L$ and $R$ for each module. The third dimensionless parameter $H$ is varied by different gap heights.

Tab. 1: Dimensionless parameters of the pocket modules.

\begin{tabular}{cccc}
\hline $\mathbf{R}$ & & $\mathbf{L}$ & \\
\hline & $\mathbf{0 . 5 0}$ & $\mathbf{0 . 6 5}$ & $\mathbf{0 . 8 0}$ \\
\hline $\mathbf{0 . 2 0}$ & $M 1$ & M2 & M3 \\
$\mathbf{0 . 5 0}$ & $M 4$ & M5 & M6 \\
$\mathbf{0 . 8 0}$ & M7 & M8 & M9 \\
\hline
\end{tabular}

Ideally, the tilting stiffness of the bearing modules is significantly higher than the calculated one of the test bench. Therefore, according to Eq. (7), all modules' outer diameters are constant at $50 \mathrm{~mm}$. As a consequence, the variation of $r_{i}$ and $r_{s}$ sets the dimensionless parameters $L$ and $R$. All modules have a nominal step height $h_{s}$ of $10 \mu \mathrm{m}$. According to Bassani, the arithmetical surface roughness $R_{a}$ should be less than 0.1 times the gap height [Bassani 1992]. Therefore, roughly machined modules of hardened and tempered 1.7225 steel were surface ground. Subsequently, the shallow recess was precision milled. The endmill used had a corner radius of $0.2 \mathrm{~mm}$, which produced a continuous transition at the step. Fig. 7 shows the finish machined and assembled module M8.

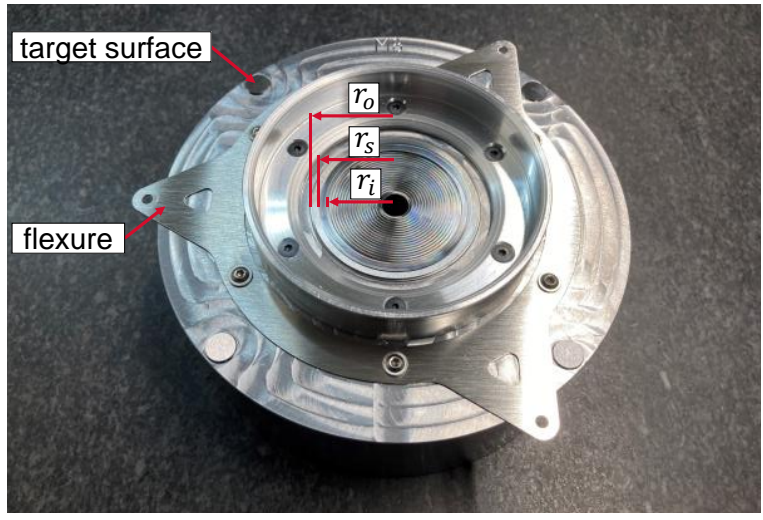

Fig. 7: Assembled module M8.

Due to the small step height, errors in the magnitude of the recess are unavoidable. Therefore, we measured the step height at several locations. Tab. 2 lists the average step height $\bar{h}_{s}$, maximum deviation in step height $\Delta h_{s}$, mean roughness on the land $R_{z, l}$ and recess $R_{z, r}$ as well as the arithmetical surface roughness at the land $R_{a, l}$ and recess $R_{a, r}$.
Tab. 2: Bearing module properties in $\mu m$.

\begin{tabular}{ccccccc}
\hline Module & $\overline{\boldsymbol{h}}_{\boldsymbol{s}}$ & $\Delta \boldsymbol{h}_{\boldsymbol{s}}$ & $\boldsymbol{R}_{\boldsymbol{z}, \boldsymbol{r}}$ & $\boldsymbol{R}_{\boldsymbol{a}, \boldsymbol{r}}$ & $\boldsymbol{R}_{\boldsymbol{z}, \boldsymbol{l}}$ & $\boldsymbol{R}_{\boldsymbol{a}, \boldsymbol{l}}$ \\
\hline M1 & 9.1 & 0.3 & 1.210 & 0.099 & 3.465 & 0.374 \\
M2 & 10.0 & 0.5 & 1.600 & 0.153 & 3.295 & 0.346 \\
M3 & 8.7 & 0.4 & 0.570 & 0.068 & 3.525 & 0.376 \\
M4 & 9.5 & 0.4 & 1.025 & 0.095 & 3.563 & 0.374 \\
M5 & 10.2 & 0.5 & 1.063 & 0.112 & 3.675 & 0.391 \\
M6 & 8.9 & 0.6 & 0.938 & 0.100 & 3.163 & 0.355 \\
M7 & 9.2 & 0.4 & 1.106 & 0.148 & 2.646 & 0.416 \\
M8 & 8.4 & 0.4 & 0.621 & 0.095 & 2.685 & 0.421 \\
M9 & 10.3 & 0.8 & 0.583 & 0.069 & 2.967 & 0.486 \\
\hline
\end{tabular}

\subsection{Test environment}

The ambient temperature in the laboratory was $20 \pm 2{ }^{\circ} \mathrm{C}$ during the experiments. However, the main body reached a maximum temperature of $23^{\circ} \mathrm{C}$ due to the throttling of pressurized oil.

\subsection{Hydraulic supply}

The hydraulic fluid was an ISO VG 15 grade oil pressurized to $10 \pm 0.05 \mathrm{MP}$. A high-pressure heat exchanger regulated its temperature to $20 \pm 2{ }^{\circ} \mathrm{C}$. In addition, Tab. 3 lists the properties of the fluid used.

Tab. 3: Properties of the fluid.

\begin{tabular}{cccc}
\hline Oil type & $\begin{array}{c}\text { Kinematic } \\
\text { viscosity } \\
\text { at } 20^{\circ} \mathrm{C}\end{array}$ & $\begin{array}{c}\text { Dynamic } \\
\text { viscosity at } \\
\mathbf{2 0} 0^{\circ} \mathrm{C}\end{array}$ & Density \\
\hline VG 15 & $28 \frac{\mathrm{mm}^{2}}{\mathrm{~s}}$ & $23 \mathrm{mPas}$ & $820 \frac{\mathrm{kg}}{\mathrm{m}^{3}}$
\end{tabular}

\section{SIMULATION}

A program in MATLAB R2018b solves Eq. (1) numerically by finite difference method. This program uses an acceptable error of $0.01 \%$ in the pressure distribution as a convergence criterion. An ideal geometry with the constant average step height $\bar{h}_{s}$ from Tab. 1 is assumed for each module. The simulation neglects elastic and thermoelastic deformations.

\subsection{Large angle}

Due to the high mechanical compliance of the preload mechanism, a constant preload $W$ can be assumed for the present gap height changes.

$W=$ const

$W=\int_{0}^{2 \pi} \int_{r_{i}}^{r_{o}} r p(r, \theta) d r d \theta$

The program solves Eq. (1) iteratively for a given angular error until Eq. (10) is satisfied. Fig. 8 shows an exemplary solution for two modules. Depending on the bearing geometry, an angular change can increase or decrease the gap height. 


\section{HSM \\ 2021 \\ MM Science Journal | www.mmscience.eu \\ ISSN 1803-1269 (Print) | ISSN 1805-0476 (Online) \\ Special Issue | HSM 2021 \\ $16^{\text {th }}$ International Conference on High Speed Machining \\ October 26-27, 2021, Darmstadt, Germany}

DOI: 10.17973/MMSJ.2021_11_2021141
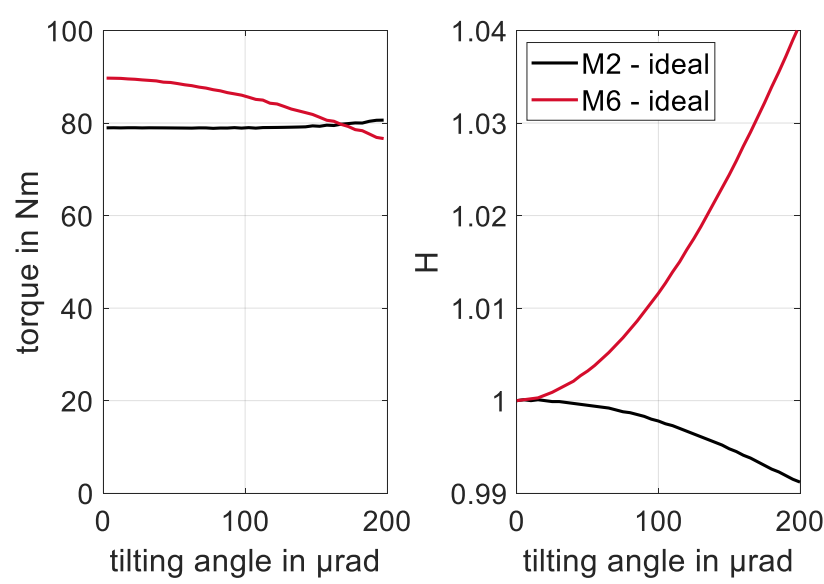

Fig. 8: Influence of tilting angle on gap height.

Additionally, a crosstalk of the moment load on the gap height exists. However, correct design parameters of the bearing geometry can minimize the effect.

\subsection{Small-angle assumption}

In the following, the study considers angular changes as small if the change in gap height over the bearing diameter is less than one-quarter of the nominal gap height. Referencing Fig. 8, this corresponds to an angle of $50 \mu \mathrm{rad}$ at a gap height of $10 \mu \mathrm{m}$.

Considering small angular changes, the assumption of constant tilting stiffness and gap height is acceptable. This simplification significantly reduces the calculation time.

\section{EXPERIMENTAL}

\subsection{Test procedure}

Both trays of the torque contraption contained equal masses and the supply pressurized the test bench. The gap height was adjusted by tightening the preloading screw. Additional weights obtained a constant moment to establish the parallel orientation of the bearing, when necessary. Before the start of the measurement, we relieved the oil supply pressure. After that, the measurement started and with a delay, the oil supply pressurized the test bench again. With this, the measurement also recorded the initia lift respectively gap height. Moving the masses to the opposite side of the contraption increased the applied torque incrementally. To avoid contact and thus damage to the bearing surfaces, the maximum tilt angle was limited. This limitation corresponds to a tilting angle of the bearing over its diameter of once the nominal gap height. After the maximum allowable tilting angle was present, the procedure reversed. At the end of the test, a moment-free condition was again present. Thus, thermal drifts, hysteresis effects or general errors were detectable. The application of the moment was in line with the displacement transducer to increase the measurement sensitivity.

Fig. 9 shows an example of the recorded data. This consists of the measured values of the displacement transducers (DT) and the calculated moment based on the mass distribution. The evaluation used the sections highlighted in gray with constant moment load. Shifting the masses between these sections excited the system and generated an intermediate step. Finally, the measured values of the displacement transducers were averaged over the evaluation area to compensate for oscillations.
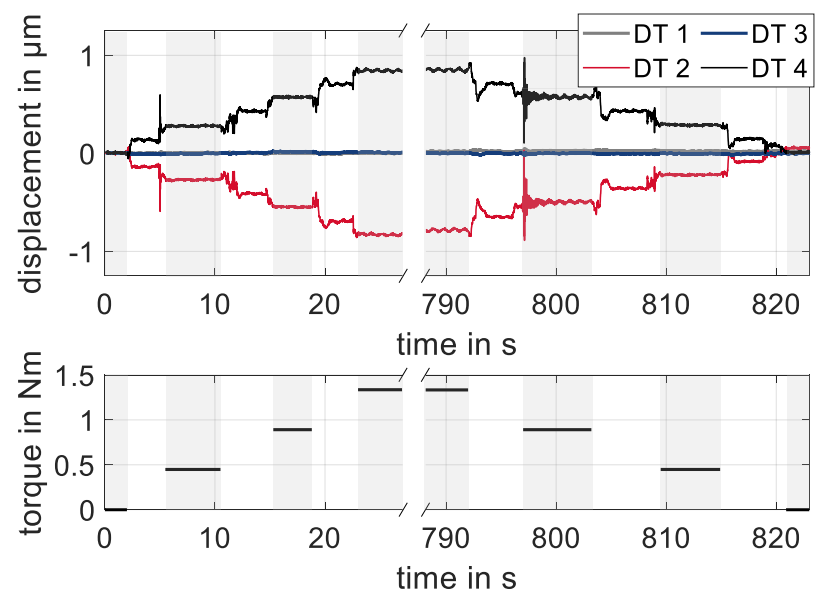

Fig. 9: Section of raw data module $M 3, H=0.99$.

Based on the four displacements, a best-fit plane is generated. A criterion for detecting measuring errors allows a maximum distance between the measuring point and plane of $0.2 \mu \mathrm{m}$. The normal vector of the plane defines the tilting angle - the gap height results from the mean value of the four displacement transducers. Finally, the derivation of the angular change represents the tilting stiffness.

Before the primary investigation of the bearing geometry, we investigated the influence of the boundary conditions.

\subsection{Influence of supply pressure}

A higher supply pressure influences the preload of the bearing and the flow conditions. We selected Module M1 to investigate the influence of the supply pressure. This module has both the lowest elastic deformation and the slightest variation in step height $\Delta h_{s}$. According to the Reynolds equation, the tilting stiffness behaves proportionally to the supply pressure. Fig. 10 shows the results for a supply pressure between 2.5 and $10 \mathrm{MPa}$.
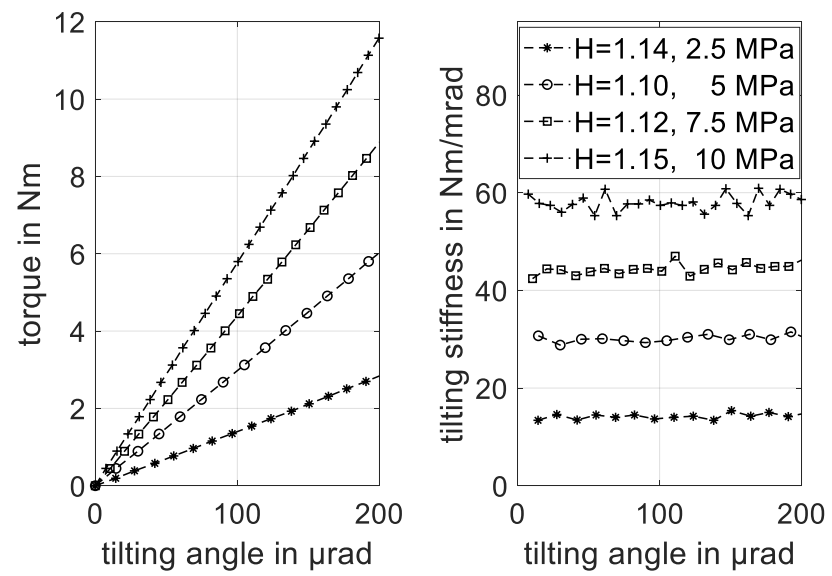

Fig. 10: Influence of supply pressure.

Considering the differences in $H$ respective the gap height $h_{g}$ at the different supply pressures, the four measurements confirm the proportional dependence. 


\subsection{Influence of load direction}

Module M9 has the highest error in step height. Due to the high preload of this module, fine adjustments of the gap height were not possible. Therefore, module M6 with the second-highest error served as the reference to determine the influence of the loading direction. The basic alignment of the microstructures of the bearing module and the main body were perpendicular to each other. The load direction of the first measurement was at $45^{\circ}$ relative to both microstructures. For each subsequent measurement, we rotated the load direction by $90^{\circ}$. This method prevents a change in microstructure. Fig. 11 shows the four measurements.
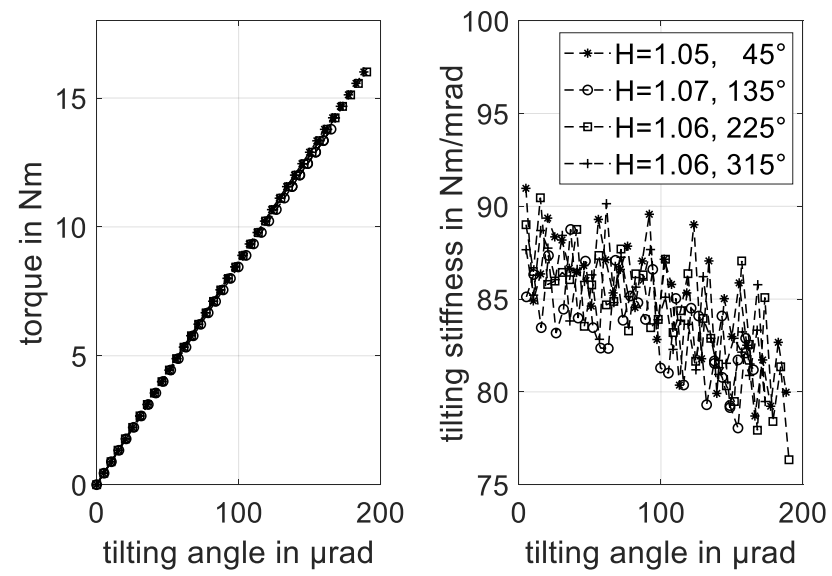

Fig. 11: Influence of load direction.

The deviation correlates with the variation of the gap height $h_{g}$. For this reason, the tilting stiffness is independent of the load direction.

\subsection{Influence of surface microstructure}

The ground surface is relatively rough compared to the gap height and the properties are directional. Due to the lowest variation of the step height and the broadest land, module M1 served as a reference. In the first measurement, the microstructure of both components was parallel $\left(0^{\circ}\right)$. In the second measurement, the microstructure was perpendicular to each other. Fig. 12 shows the results.
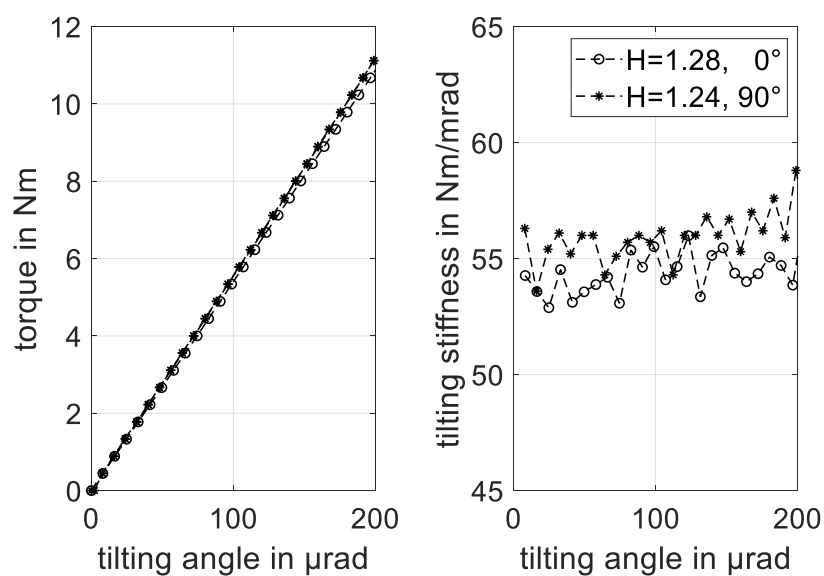

Fig. 12: Influence of surface microstructure.

The deviation correlates with the variation of the gap height $h_{g}$. For this reason, the tilting stiffness is independent of the surface microstructure orientation.

\subsection{Influence of bearing geometry}

Based on the preliminary tests performed, a constant supply pressure of $10 \mathrm{MPa}$ was selected. Since there is no dependence on the orientation or microstructure, performing the tests for one load direction is sufficient. The incremental loading and unloading of the torque contraption enable the detection of the hysteresis.

Fig. 13 summarizes all measurements of modules M1- M9. For each module, the plot shows the measured tilting angle against torque. For a better overview, each graph includes a sketch of the corresponding bearing geometry. Here, the gray areas correspond to the recess and the black areas to the land. The adjacent graph shows the calculated stiffnesses. In addition, this graph contains the simulated tilting stiffnesses for comparison. As described in section 4.2 , the measurements validate the assumption of constant stiffness for small angles. Tab. 4 lists the measured tilting stiffnesses for small angles. The percentages in the parentheses indicate the deviation of the measured values from the simulation. Exact values for $H$ can be taken from Fig. 13.

Tab. 4: Measured small angle tilting stiffness in $\mathrm{Nm} / \mathrm{mrad}$ and deviation to the simulation.

\begin{tabular}{ccccc}
\hline $\mathbf{H}$ & $\mathbf{R}$ & & $\mathbf{L}$ & $\mathbf{0 . 6 5}$ \\
\hline \multirow{3}{*}{$(\mathbf{0 . 7 5 )}$} & & $\mathbf{0 . 5 0}$ & $83.4(-8.0 \%)$ & $130.9(-10.3 \%)$ \\
& $\mathbf{0 . 2 0}$ & $77.5(-7.6 \%)$ & $67.4(-3.3 \%)$ & $108.7(-11.3 \%)$ \\
& $\mathbf{0 . 5 0}$ & $54.7(-2.3 \%)$ & $41.0(+8.6 \%)$ & $48.8(+3.0 \%)$ \\
\hline \multirow{2}{*}{$(\mathbf{1 . 0 0 )}$} & $\mathbf{0 . 8 0}$ & $23.5(+6.3 \%)$ & $71.2(-9.3 \%)$ & $104.3(-12.9 \%)$ \\
& $\mathbf{0 . 2 0}$ & $64.2(-9.2 \%)$ & $59.8(-4.3 \%)$ & $90.9(-9.0 \%)$ \\
& $\mathbf{0 . 5 0}$ & $48.5(-4.3 \%)$ & $35.6(+5.0 \%)$ & $41.9(+6.9 \%)$ \\
\hline \multirow{2}{*}{$(\mathbf{1 . 2 5 )}$} & $\mathbf{0 . 8 0}$ & $21.5(+5.0 \%)$ & $62.2(-8.1 \%)$ & $86.0(-8.5 \%)$ \\
& $\mathbf{0 . 2 0}$ & $55.4(-8.1 \%)$ & $52.4(-3.7 \%)$ & $76.6(-5.7 \%)$ \\
& $\mathbf{0 . 5 0}$ & $42.8(-3.7 \%)$ & $30.4(+3.1 \%)$ & $35.6(+7.2 \%)$ \\
\hline
\end{tabular}



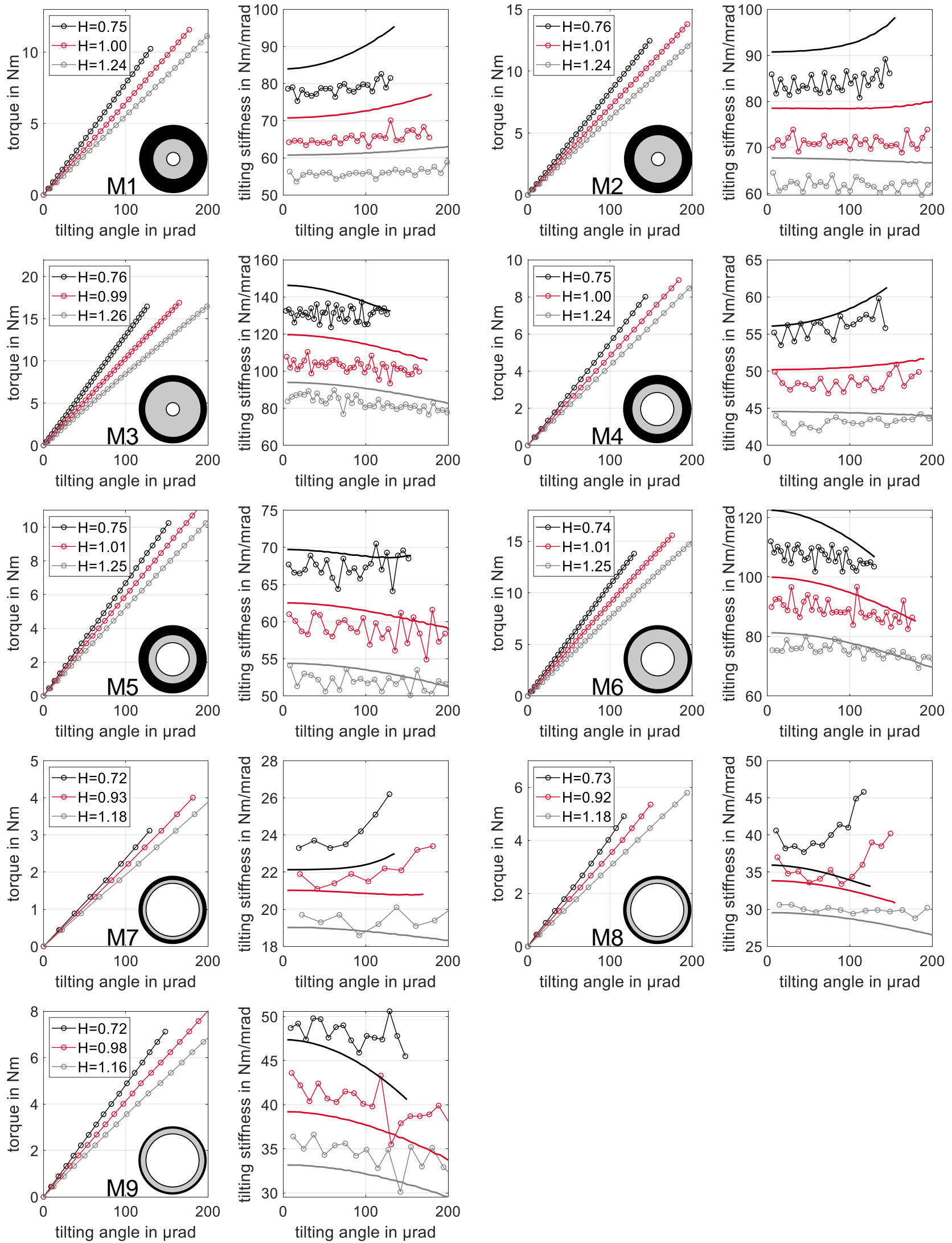

Fig. 13: Measured (dotted lines) tilting angle under torque and calculated tilting stiffness compared to the simulation (continuous lines) of modules M1 - M9. 


\section{HSM \\ 2021}

MM Science Journal | www.mmscience.eu

ISSN 1803-1269 (Print) | ISSN 1805-0476 (Online)

Special Issue | HSM 2021

$16^{\text {th }}$ International Conference on High Speed Machining

October 26-27, 2021, Darmstadt, Germany

DOI: 10.17973/MMSJ.2021_11_2021141

Independent of the height ratio $H$, respectively the gap height $h_{g}$, module M3 shows the highest tilting stiffness. In general, a high value for $L$ and a small value for $R$ provide a high tilting stiffness. Since the gap height changed the height ratio, the increase in tilting stiffness is not exclusively due to the height ratio. Considering Eq. (7), the influence of the gap height change and the height ratio can be separated mathematically. The influence of the gap height change is dominant.

When comparing the measured and simulated tilting stiffness, a correlation with $R$ exists. The simulation underestimates the tilting stiffness for high $R$ values and overestimates for small ones. Mainly responsible for this behavior are elastic deformations. The area on which the supply pressure acts is proportional to $R$. Therefore, the preload and deformation are greater for bearings with high $R$. As a result, the measured values of the displacement transducers overestimate the actual gap height.

Another correlation exists with the variation of the step height. Modules M6 and M9 with the highest variation show lower tilting stiffness relative to the simulation than the other modules with identical $R$ values. This effect increases with smaller gap heights.

For small $L$ values, the modules show an increase in tilting stiffness with increasing tilting angles. Conversely, for large $L$ values, the tilting stiffness decreases. This effect is more pronounced for low gap heights and independent of $R$. The behavior corresponds to the assumption of constant preload.

\section{SUMMARY}

An experimental study on the tilting stiffness of shallow recess oil hydrostatic thrust bearings was conducted. For this, the test series experimentally investigated the tilting stiffness of different bearing geometries. The presented test bench allowed repeatable measurements with negligible hysteresis. In addition, numeric calculations based on the 2D-Reynolds equation show a high correlation with the measured values. Particularly at high preloads, elastic deformations can influence tilting stiffness depending on the bearing geometry, machine structure, supply pressure and gap height.

\section{REFERENCES}

[Adams 1958] Adams, C.R. The STEP BEARING - a new concept in air lubrication. Product engineering, December 1958, Vol.29, No.50, pp 46-53. ISSN 0032-9754

[Bassani 1992] Bassani, R. and Piccigallo, B. Hydrostatic Lubrication. Elsevier Science, August 1992. ISBN 9780444884985

[Beek 2019] A. Van Beek. Advanced Engineering Design: Lifetime Performance and Reliability. TU Delft, 2019. ISBN 9789081040617

[Carpenter 1969] Carpenter, A.L. An investigation into the characteristics of tapered and stepped land hydrostatic journal bearings. Adelaide: University of Adelaide, Department of Mechanical Engineering, 1969.

[Donaldson 1965] Donaldson, R.R. Incompressible journal bearings with combined hydrostatic-hydrodynamic action. Massachusetts: Massachusetts Institute of Technology, Department of Mechanical Engineering, 1965.

[Knapp 2019] Knapp, B., et al. Modeling dynamic stiffness of an air bearing spindle using FRFs. Annual Meeting of the American Society for Precision Engineering, Pittsburgh, October 2019.

[Liu 2017] Liu, Z., et al. A review of hydrostatic bearing system: Researches and applications. Advances in Mechanical Engineering, October 2017, Vol.9, No.10. ISSN 1687-8140

[Pollmann 1989] Pollmann, E. and Vermeulen, M. Compressibility and inertia effects on the dynamic behavior of recessed hydrostatic bearings. Tribology International, June 1989, Vol.22, No.3, pp 166-176. ISSN 0301-679X

[Rowe 2012] Rowe, W.B. Hydrostatic, Aerostatic and Hybrid Bearing Design. Butterworth-Heinemann, 2012. ISBN 9780123969941

[Schlegel 2018] Schlegel, C., et al. Metrological Characterization of a $20 \mathrm{Nm}$ Torque Calibration Standard Machine at PTB, Germany. Journal of Physics: Conference Series, 2018, Vol.1065, No.4. ISSN 1742-6596 